TITLE:

\title{
Phase synchronization of autonomous AC grid system with passivity-based control
}

$\operatorname{AUTHOR}(\mathrm{S})$ :

Manohar, Rutvika; Hikihara, Takashi

\section{CITATION:}

Manohar, Rutvika ...[et al]. Phase synchronization of autonomous AC grid system with passivity-based control. International Journal of Circuit Theory and Applications 2020, 48(6): 906-918

\section{ISSUE DATE:}

2020-06

URL:

http://hdl.handle.net/2433/251421

\section{RIGHT:}

This is the peer reviewed version of the following article: Manohar, R, Hikihara, T. Phase synchronization of autonomous AC grid system with passivity - based control. Int J Circ Theor Appl. 2020; 48: 906- 918, which has been published in final form at https://doi.org/10.1002/cta.2760. This article may be used for non-commercial purposes in accordance with Wiley Terms and Conditions for Use of Self-Archived Versions.; The full-text file will be made open to the public on 4 March 2021 in accordance with publisher's 'Terms and Conditions for Self-Archiving'.: この論文は出版社版でありまサ ん。引用の際には出版社版をご確認ご利用ください。; This is not the published version. Please cite only the published version. 


\title{
Phase synchronization of autonomous AC grid system with passivity-based control
}

\author{
Rutvika Manohar* and Takashi Hikihara ${ }^{\dagger}$ \\ Department of Electrical Engineering, Kyoto University, Katsura, Nishikyoku, Kyoto, Japan 615-8510
}

(Dated: March 23, 2020)

\begin{abstract}
This paper discusses a ring coupled buck-type inverter system to harness energy from DC sources of electricity. The DC-DC buck converter circuit is modified with an H-bridge to convert the DC input voltage to a usable $\mathrm{AC}$ output voltage. Passivity-based Control (PBC) with Port Controlled Hamiltonian Modelling ( $\mathrm{PCHM}$ ) is a method where the system is controlled by considering not only the energy properties of the system but also the inherent physical structure. PBC is applied to achieve stabilization of the AC output voltage to a desired amplitude and frequency. Unsynchronized output voltages in terms of phase angle or frequency can cause detrimental effects on the system. PLL is employed in the ring structure to maintain synchronization of the AC output voltage of all inverter units in the ring coupled system.
\end{abstract}

\section{INTRODUCTION}

Harnessing renewable energy for the production of electricity is seen as one of the most important task for researchers in the field of electrical engineering today. Generation and storage of electricity with non-conventional power sources like photovoltaic cells, fuel cells, wind power, and battries is realized with distributed generation [1,2]. With the increase in the amount of renewable energy available, the integration of renewable energy to the existing power supply mechanism is imperative $[3,4]$.

Distributed generation or dispersed generation is defined as electric power generation within distribution network, or on the consumer side of the network [1]. It consists of generation and storage of electricity with the help of various distributed resources (power resources covering a large area) connected in a grid. An autonomous distributed generation system is dispersed generation not connected to the conventional electric grid. A distributed power generation system can be used to harness energy from AC as well as DC sources of electricity. Examples include stand-alone power grids in rural areas and microgrids and smart grids in urban areas [5-7]. To ensure stable operation of a distributed generation system, there is a growing need for a control stratergy which will be able to incorporate the diverse nature of renewable energy resources and increase operational flexibility $[8,9]$.

In this paper we focus on renewable DC sources of electricity, and suggest an autonomous grid formulation to utilize the DC electric power, motivated by recent stand-alone solar power initiatives in rural areas in various developing countries $[6,10,11]$. We introduce a ring coupled buck-type inverter system to utilize energy from DC power sources by converting it to AC voltage. The basic design of this grid is shown in Fig.1. In the ring coupled buck-type inverter system, multiple units of inverter circuits are connected in a ring formulation with dissipation in between them. Power inverters play the role of an interface to transfer energy from DC sources of electricity to AC voltage form with a desired frequency $[12,13]$. The buck-type inverter circuit employs the circuit configuration of a DC/DC buck converter with the DC input switched using a H-bridge circuit which enables to change the polarity to obtain AC output voltage.

The stabilization of the grid to the desired AC voltage, usually carried out by AVR and governer, is replaced by passivity-based control (PBC). This method of control focuses on the energy characteristics of the autonomous grid [14]. PBC is employed for passive systems, i.e systems where energy stored in the system is always less than the energy supplied to the system from outside [15] and is performed in two stages. The first stage entails modifying the energy of the system such that the modified energy function has a unique global equilibrium at the desired state, satisfying the passification objective. It is referred to as energy-shaping. The second stage is to add damping to make the system exponentially stable [16]. In this way, PBC forces individual inverter units to share energy so as to allow the entire system to retain passivity and achieve stability at the desired state.

Port Controlled Hamiltonian Modelling (PCHM) is an indespensable tool for applying PBC to electrical systems that have switching elements and dissipation [17]. It facilitates the description of the system into well defined matrices indicating dissipation, structure and external inputs to the system. Hamiltonian modelling for optimal control of converters has been shown [18]. In this paper we implement PCHM to the autonomous AC grid formulate the control rule using PBC.

\footnotetext{
*r-manohar@dove.kuee.kyoto-u.ac.jp

$\dagger$ hikihara.takashi.2n@kyoto-u.ac.jp
} 


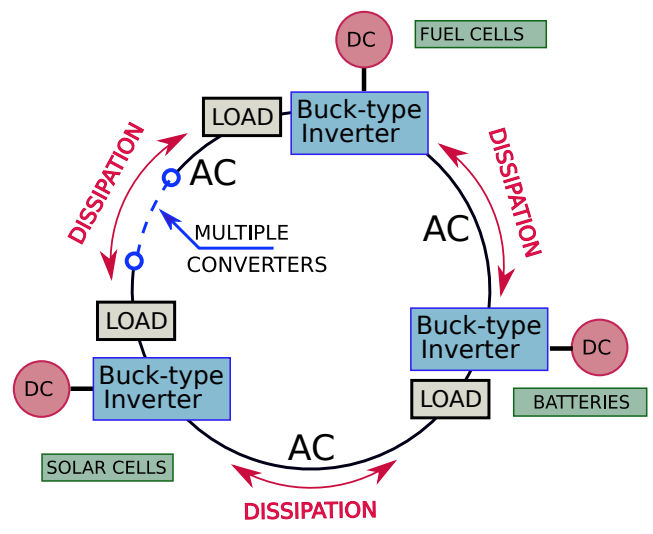

FIG. 1: Proposed autnomous AC grid.
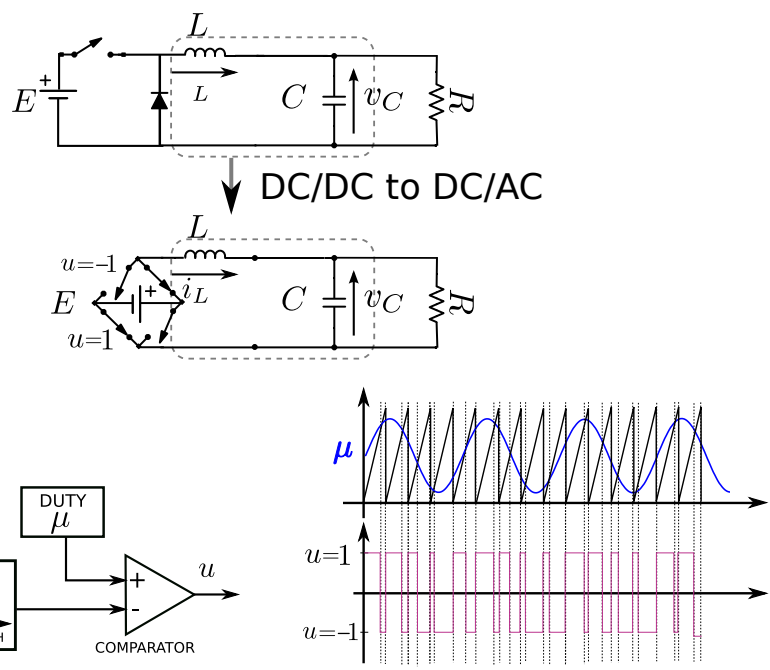

FIG. 2: Working of the buck-type inverter.

The application of PBC for DC/DC converters was shown in [19]. Analysis of the transient behaviours of boost converters with respect to the energy characteristics was performed in [20]. Then, the application of PBC for parallely connected DC/DC converters was described in detail in [21]. In both the above mentioned works, the objective was to stabilize the system to a constant DC voltage. When stabilizing the system to a sinusoidal desired state, the steady state analysis is significantly intricate. This paper lays out the steady state analysis as well as formulation of the control equations for a sinusoidal desired state.

Another crucial task is that of grid synchronization. Existing projects of stand-alone PV systems have often reported problems of grid stability due to lack of synchroinization [9, 22]. Various methods for synchronizing the power from multiple renewable resources to the existing power system are shown in [23]. Here, we focus on synchronizing the multiple AC voltage outputs to the desired state in terms of frequency and phase. It is shown that, if the multiple inverters in the ring coupling have inconsistent phase or frequency, it can prove to be harmful to the system. It is desirable that all the inverters autonomously adjust themselves to the desired sinusoidal state without any phase difference. To achieve this, phase synchronization for all the inverter units in the grid is implemented with attached phase locked loop (PLL) [24].

Finally, the theory is successfully tested with numerical simulations to illustrate the temporal behaviour of the system. Numerical simulations are performed in the SIMULINK environment on MATLAB R2017.

\section{BUCK-TYPE INVERTER}

The circuit configuration of the buck-type inverter is shown in Fig.2. A double bridge is implemented to invert the polarity of the DC source []. Here, $u$ represents the instantaneous switch position as shown in the figure. The instantaneous switch position takes the values in the discrete set $\{-1,1\}$ as shown in the figure. It is seen that the value of $u$ is responsible for changing the polarity of the DC source, thus making it possible to obtain the negative part of the sinusoidal output voltage.

To obtain a constant $\mathrm{AC}$ output voltage, the control objective is to regulate the average output voltage to a periodic value with frequency much smaller than the switching frequency (usually 50 or $60 \mathrm{~Hz}$ ). Then, it is desirable to consider the average value of the voltages and currents rather than the instantaneous values, given that the ripple and harmonics are sufficiently small. To derive an averaged model, it is necessary to average the circuit variables as well as the switching function [12]. Hence, we replace the instantaneous switch position with a modulating signal for the averaged circuit. Hereafter, the modulating function will be referred to with the variable $\mu(t)$.

To implement a PWM policy for switch regulation for generating a digital pulse signal to drive the transistor switches, this modulating function $\mu$ acts as the continuous control input. As the modulating signal varies slowly in comparison to the frequency of the switching, it can be shown that the switching function is equal to the modulating signal [12]. We refer to the switching function as the duty ratio.

To obtain the averaged circuit variables, we replace the instantaneous values by the average values. Thus, $i_{L}$ and $v_{C}$ are the average the inductor current and capacitor voltage respectively, and $\mu$, the duty ratio. The output voltage of the inverter depends on the duty cycle of the switching [25]. Thus, the averaged control input is takes values in 
the closed set $[-1,1]$. The averaged duty cycle will be sinusoidally modulated to achieve a sinusoidal output voltage. The average model of a buck-type inverter is given in Eq.(1). Owing to the buck-type configuration, the peak output voltage will be less than the DC input voltage.

$$
\begin{aligned}
L \dot{i}_{L} & =-v_{C}+\mu E \\
C \dot{v}_{C} & =i_{L}-v_{C} / R
\end{aligned}
$$

Here $L, C$ and $R$ represents the inductance, capacitance and load resistance respectively. $E$ is the external DC input. Optimal control of DC/AC inverters with an H-bridge topology has been obtained in [13]. Here, we aim to stabilize the output of the inverter with PBC.

\section{SYSTEM DESIGN}

The AC grid has multiple DC sources each connected to a buck-type inverter, to invert the DC voltage to AC output, which can be used by the existing household loads. These inverters are coupled together with dissipation through inductance and resistance.

The schematic diagram of the circuit is shown in Fig. 3. The role of the control input, for each buck-type inverter, is to decide the amplitude and frequency of the output voltage. In a ring coupled configuration, it is imperative that the output voltage of each inverter converge to the same value. Then, the voltage in the ring will be maintained at a user-defined desired value.

Here, load resistances $\left(R_{2 T}\right)$ are across the output voltages whereas the dissipation elements of line inductor $\left(L_{t}\right)$ and line resistance $\left(R_{1 T}\right)$ are in series. The coupling through dissipation represents a transmission line model, with inductive and resistive elements. The capacitor in the transmission line model is considered as negligible. This is owing to the fact that a parallel capacitor is dominant in the buck-type inverter configuration. The number of inverters in the ring does not cause any loss of generality. Then, the objective is to apply PBC to the whole system, including the dissipation between the inverters.

$$
\begin{aligned}
L_{\mathrm{n}} \frac{d i_{L_{\mathrm{n}}}}{d t} & =-v_{C_{\mathrm{n}}}+\mu_{\mathrm{n}} E_{\mathrm{n}} \\
C_{\mathrm{n}} \frac{d v_{C_{\mathrm{n}}}}{d t} & =i_{L_{\mathrm{n}}}-i_{T \mathrm{n}}+i_{T \mathrm{n}-1}-\frac{v_{C_{\mathrm{n}}}}{R_{2 \mathrm{n}-1}} \\
L_{T \mathrm{n}} \frac{d i_{T \mathrm{n}}}{d t} & =v_{C_{\mathrm{n}}}-v_{C_{\mathrm{n}+1}}-R_{1 \mathrm{n}} i_{T \mathrm{n}}
\end{aligned}
$$

The averaged model of the ring coupled buck-type inverter system is given by Eqs.(2)-(4). Subscription $n$ denotes the index of the inverter. $\mu_{\mathrm{n}}$ denotes duty ratio function for the $\# \mathrm{n}$ inverter. $L_{T \mathrm{n}}$ is the inductance and $R_{1 T \mathrm{n}}$ in the dissipation between the $\# 0$ and $\# 1$ inverter. The circuit variables, $v_{C n}, i_{L n}$ and $i_{T n}$ denote the average of the instantaneous value over one switching cycle, as the frequency of these variables is much smaller than the switching frequency.

To ensure phase and frequency synchronization of the ring coupled inverter system, PLLs are added to the basic circuit formulation. Let $\bar{v}_{C \mathrm{n}}=A_{n} \sin \left(\Phi_{\mathrm{n}}\right)$ be the desired sinusoidal output voltage. Then, $\sin \left(\Phi_{\mathrm{n}}\right)$ is the sinusoidal reference, where, $\Phi_{\mathrm{n}}=\omega_{d} t+\theta_{i \mathrm{n}}(t)$ is the phase. Here $\omega_{d}$ is the centre frequency, and $\theta_{i}$ the phase angle of the input singal. In order to synchronize the phase of the output voltages of ring coupled buck-type inverter systems, any \#p inverter can be taken as a reference. The sinusoidal reference for the \#p inverter is used as the input signal for the PLL, the output of which is used as the sinusoidal reference of the $\# \mathrm{p}-1$ and $\# \mathrm{p}+1$ inverter. The sinusoidal reference of $\# \mathrm{p}-1$ and $\# \mathrm{p}+1$ is in turn used as the input to PLL, the output of which is used as the sinusoidal reference to $\# \mathrm{p}-2$ and $\# \mathrm{p}+2$, respectively. It ensures minimun time delay in synchronising the phase and frequency of all the inverters in the ring. The connection of the PLL to the system is shown in Fig.4.

We consider the centre frequency $\omega_{d}$ to be the same for all the PLLs employed in the ring coupled AC grid. Then, $\theta_{i}(t)$ incorporates the error in the input frequency $\left(\omega_{i}\right)$ from the centre frequency of the voltage controlled oscillator (VCO). This error is given by $\Delta \omega=\omega_{i}-\omega_{d}$. Let the output of the VCO be $r\left(t, \tilde{\Phi}_{\mathrm{n}}\right)=V_{0} \cos \left(\omega_{d} t+\theta_{o}(t)\right)$, with $V_{0}$ as the amplitude and $\tilde{\Phi}=\omega_{d} t+\theta_{o}(t)$ as the phase. $\theta_{o}$ represents the phase angle of the output signal. The state equation governing the change in the phase are given by Eq.(34) [26, 27].

$$
\frac{d \theta_{o}}{d t}=K_{C}\left[\mathcal{L}^{-1}\left[F(s) V_{\text {comp }}(s)\right] K_{p} A \sin \left(\theta_{e}\right)+v_{e}\right]
$$

Here, $\theta_{e}=\theta_{i}-\theta_{o}$ is the phase error, $v_{\text {comp }}$ is the input of the low pass filter, and $V_{\text {comp }}(s)$ is the Laplace transform of $v_{\text {comp }}$, which is the output voltage of the comparator. The transfer function of the LPF in the frequency domain 


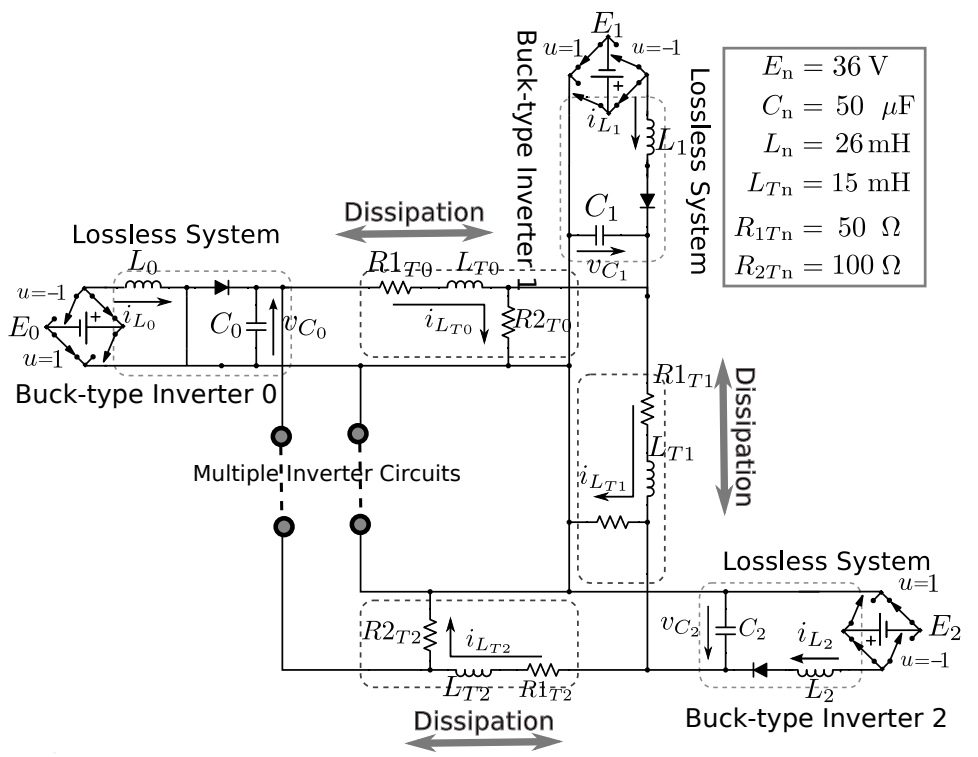

FIG. 3: Schematic circuit diagram of ring coupled buck-type inverters.

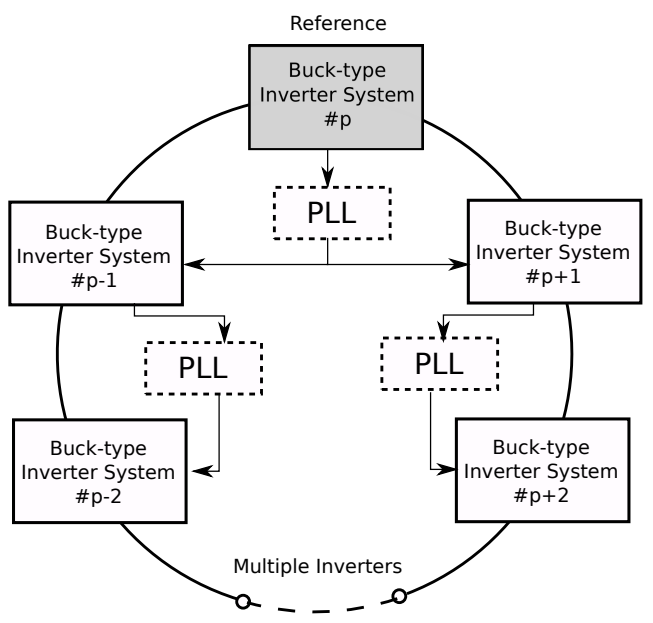

FIG. 4: System configuration: Connection of PLL to multiple inverter system.

is given as $F(s)$ and $K_{p}$ and $K_{C}$ are the gains of the phase detector and VCO of the PLL respectively. Lastly, $\mathcal{L}^{-1}$ denotes the Laplace inverse and $v_{e}$ is the external control voltage. For more details on the working of PLL, please refer to Appendix A.

\section{PORT CONTROLLED HAMILTONIAN MODELLING}

Port Controlled Hamiltonian Modeling(PCHM) is the network representation of systems in interaction with their environment [17]. The Hamiltonian approach allows for the systematic modelling of electrical systems including resistors and switching elements. The non-energetic terms are extracted from the circuit. It leaves the energy conserving LC circuit. The non-energy elements are then introduced into the circuit in the form of 'ports' [17]. Such configuration of LC circuits with ports is represented as a generalized Hamiltonian system with external input variables. PCHM technique is used for modeling the inverter circuit, to allow for the inclusion of power electronic switches and load resistances.

PCHM classifies the system neatly into physically well defined interconnection $(\mathbf{J})$, dissipation $(\mathbf{R})$ and external in$\operatorname{put}(\mathbf{E})$ matrices within a state space framework [16]. The system model using the Port Controlled Hamiltonian $(\mathrm{PCH})$ framework is given by Eq.(7) as in the form given in [28].

$$
\begin{aligned}
\mathbf{D} \dot{\boldsymbol{x}}(t) & =[\mathbf{J}-\mathbf{R}] \frac{\partial H_{e}}{\partial x}+\mathbf{G}(\boldsymbol{x}) \mathbf{E} \\
\boldsymbol{y} & =\mathbf{G}^{T}(\boldsymbol{x}) \frac{\partial H_{e}}{\partial \boldsymbol{x}}
\end{aligned}
$$

For $\mathrm{m}$ inverters in the configuration, $\boldsymbol{x}$, the state of the system, is a column matrix $((\mathrm{m} \times 3) \times 1)$ of all the inductor currents and capacitance voltages. The matrix $\mathbf{D}$, is a diagonal matrix of the capacitances and inductances of the corresponding currents and voltages. $\mathbf{J}$ and the vector function $\mathbf{G}(\boldsymbol{x})$ gives the interconnection, and $\mathbf{R}$ the dissipation in the system. $\mathbf{G}(\boldsymbol{x})$ is a function of $u_{\mathrm{n}}$, the switch position of the corresponding buck-type inverters in the ring configuration. $\mathbf{E}$, the input matrix is a column matrix of the input voltages to the respective inverters. $H_{e}=\frac{1}{2} \boldsymbol{x} I \boldsymbol{x}$ is the Hamiltonian. Here, $I$ is the identity matrix.

Here, $\mathbf{D}, \mathbf{R}, \mathbf{J}$ and $\mathbf{G}$ are $((\mathrm{m} \times 3) \times(\mathrm{m} \times 3))$ matrices, $\mathbf{E}$ is a $(\mathrm{m} \times 3) \times 1$ matrix.

$$
\mathbf{D}=\left[\begin{array}{cccc}
\mathbf{D}_{0} & 0 & \ldots & 0 \\
0 & \mathbf{D}_{1} & \ldots & 0 \\
\vdots & \vdots & \ddots & \vdots \\
0 & 0 & \ldots & \mathbf{D}_{\mathrm{m}}
\end{array}\right] \text { where, } \mathbf{D}_{\mathrm{n}}\left[\begin{array}{ccc}
L_{\mathrm{n}} & 0 & 0 \\
0 & C_{\mathrm{n}} & 0 \\
0 & 0 & L_{T \mathrm{n}}
\end{array}\right] \text {, and } \boldsymbol{x}=\left[\begin{array}{c}
\boldsymbol{x}_{0} \\
\vdots \\
\boldsymbol{x}_{\mathrm{m}}
\end{array}\right] \text { where, } \boldsymbol{x}_{\mathrm{n}}=\left[\begin{array}{c}
i_{L \mathrm{n}} \\
v_{C \mathrm{n}} \\
i_{T \mathrm{n}}
\end{array}\right] \text {. }
$$




$$
\begin{aligned}
& \mathbf{J}=\left[\begin{array}{cccccc}
\mathbf{A}_{0} & -\mathbf{B}^{T} & 0 & \ldots & 0 & \mathbf{B} \\
\mathbf{B} & \mathbf{A}_{1} & -\mathbf{B}^{T} & 0 & \ldots & 0 \\
0 & \mathbf{B} & \ddots & \ddots & \ddots & \vdots \\
\vdots & 0 & \ddots & \ddots & \ddots & 0 \\
0 & \vdots & \ddots & \ddots & \ddots & -\mathbf{B}^{T} \\
-\mathbf{B}^{T} & 0 & \ldots & 0 & \mathbf{B} & \mathbf{A}_{\mathrm{m}}
\end{array}\right] \text { where, } \mathbf{A}_{\mathrm{n}}=\left[\begin{array}{ccc}
0 & -1 & 0 \\
1 & 0 & -1 \\
0 & 1 & 0
\end{array}\right], \mathbf{B}=\left[\begin{array}{lll}
0 & 0 & 0 \\
0 & 0 & 1 \\
0 & 0 & 0
\end{array}\right] \\
& \mathbf{R}=\left[\begin{array}{cccc}
\mathbf{R}_{0} & 0 & \ldots & 0 \\
0 & \mathbf{R}_{1} & \ldots & 0 \\
\vdots & \vdots & \ddots & \vdots \\
0 & 0 & \ldots & \mathbf{R}_{\mathrm{m}}
\end{array}\right] \text {, where } \mathbf{R}_{\mathrm{n}}=\left[\begin{array}{ccc}
0 & 0 & 0 \\
0 & \frac{1}{R_{2 \mathrm{n}}} & 0 \\
0 & 0 & R_{1 \mathrm{n}}
\end{array}\right] \\
& \mathbf{G}=\left[\begin{array}{cccc}
\mathbf{G}_{0} & 0 & \ldots & 0 \\
0 & \mathbf{G}_{1} & \ldots & 0 \\
\vdots & \vdots & \ddots & \vdots \\
0 & 0 & \ldots & \mathbf{G}_{\mathrm{m}}
\end{array}\right] \text {, where } \mathbf{G}_{\mathrm{n}}=\left[\begin{array}{ccc}
\mu_{\mathrm{n}} & 0 & 0 \\
0 & 0 & 0 \\
0 & 0 & 0
\end{array}\right] \text {, and } \mathbf{E}=\left[\begin{array}{c}
\mathbf{E}_{0} \\
\vdots \\
\mathbf{E}_{\mathrm{m}}
\end{array}\right] \text { where, } \mathbf{E}_{\mathrm{n}}=\left[\begin{array}{c}
E_{\mathrm{n}} \\
0 \\
0
\end{array}\right]
\end{aligned}
$$

Looking at the adjacency matrix of the interconnection matrix $(\mathbf{J})$, the ring coupled structure is clearly verified. It is seen that $\mathbf{D}$ is a diagonal matrix and $\mathbf{R}$ is a symmetric matrix for $m$ inverters. The matrix $\mathbf{J}$ is the interconnection matrix, and shows the coupling between neighbouring inverters through dissipation. From Eq.(9), it can be verified that $\mathbf{J}$ is a skew-symmetric matrix, with keeping the PCHM structure [28].

\section{STEADY STATE ANALYSIS}

To gain insight into the steady state behaviour of the system, it is desirable to establish the relationship between the equilibrium values of the average output voltage, the average input current and the average dissipation current. Then, a sinusoidal duty ratio can be implemented to obtain the desired output voltage. It is analogous to setting a constant average duty ratio for the switching for DC/DC converters. This constitues the open loop system, without a feedback control.

For the $\# \mathrm{n}$ buck-type inverter in the, the desired voltage is a $\mathrm{AC}$ waveform in the form $\bar{v}_{C \mathrm{n}}=\sin \left(2 \pi f_{\mathrm{n}} t\right)=$ $A_{\mathrm{n}} \sin \left(\Phi_{\mathrm{n}}\right) \cdot \Phi_{\mathrm{n}}=2 \pi f_{\mathrm{n}} t+\theta_{i \mathrm{n}}$ with angular frequency $\omega_{d \mathrm{n}}=2 \pi f_{\mathrm{n}}$. For steady state analysis, $\theta_{i \mathrm{n}}$ is a constant, i.e the angular frequency does not change with time.

Eqs.(2)-(4) give the mathematical description of the system for any \#n buck-type inverter system. Steady sate analysis is employed to formulate the equilibrium values for the desired inductor current $\left(\bar{i}_{L \mathrm{n}}\right)$ and the desired disspiation current $\left(\bar{i}_{T \mathrm{n}}\right)$ for a given desired voltage $v_{C \mathrm{n}}=\bar{v}_{C \mathrm{n}}$. Then, it will be possible to formulate the sinusoidal duty ratio $U$.

It is possible to analytically solve the linear differential equation Eq.(4), by employing the known values of $v_{C \mathrm{n}}=\bar{v}_{C \mathrm{n}}$, $v_{C n+1}=\bar{v}_{C n+1}$. The solution to the equation is given by Eq.(14).

$$
\bar{i}_{T \mathrm{n}}=\frac{R_{1 \mathrm{n}} A_{n}}{R_{1 \mathrm{n}}^{2}+L_{T \mathrm{n}}^{2} \omega_{d \mathrm{n}}}\left(\sin \Phi_{\mathrm{n}}-\frac{\omega_{\mathrm{n}} L_{T \mathrm{n}}}{R_{1 \mathrm{n}}} \cos \Phi_{\mathrm{n}}\right)-\frac{R_{1 \mathrm{n}} A_{n+1}}{R_{1 \mathrm{n}}^{2}+L_{T \mathrm{n}}^{2} \omega_{d \mathrm{n}+1}}\left(\sin \Phi_{\mathrm{n}+1}-\frac{\omega_{d \mathrm{n}+1} L_{T \mathrm{n}}}{R_{1 \mathrm{n}}} \cos \Phi_{\mathrm{n}+1}\right)+k e^{-\left(R_{1 \mathrm{n}} / L_{\mathrm{n}}\right) t}
$$

Here, $k$ is the constant of integration. The value of $k$ is obtained by applying the initial condition $i_{T d}(0)=0$.

$$
k=\frac{A_{\mathrm{n}} \omega_{\mathrm{n}} L_{T \mathrm{n}}}{R_{1 \mathrm{n}}^{2}+L_{\mathrm{n}}^{2} \omega_{d \mathrm{n}}}-\frac{A_{\mathrm{n}+1} \omega_{d \mathrm{n}+1} L_{T \mathrm{n}}}{R_{1 \mathrm{n}}^{2}+L_{\mathrm{n}}^{2} \omega_{d \mathrm{n}+1}}
$$

Then, the equilibrium value of $i_{L \mathrm{n}}$ can be obtained by solving Eq.(2) with $i_{T \mathrm{n}}=\bar{i}_{T \mathrm{n}}$. 


$$
\bar{i}_{L \mathrm{n}}=C_{\mathrm{n}} \dot{\bar{v}}_{C}-\bar{i}_{T \mathrm{n}}+\bar{i}_{T \mathrm{n}-1}+\frac{\bar{v}_{C}}{R_{2 \mathrm{n}-1}}
$$

Finally, the open loop duty ratio $U_{\mathrm{n}}$ to obtain from Eq.(3) by substituting $i_{L \mathrm{n}}=\bar{i}_{L \mathrm{n}}$ and given in (15).

$$
U_{\mathrm{n}}=\frac{L_{\mathrm{n}}}{E_{\mathrm{n}}}\left[C_{\mathrm{n}} \ddot{\bar{v}}_{C \mathrm{n}}-\dot{i}_{T d \mathrm{n}}+\dot{\bar{i}}_{T \mathrm{n}-1}+\frac{\dot{\bar{v}}_{C}}{R_{2 \mathrm{n}-1}}\right]+\frac{\bar{v}_{C}}{E_{\mathrm{n}}}
$$

Here, $\bar{x}$ denotes the desired averaged value, $\dot{x}$ denotes $\frac{\mathrm{d} x}{\mathrm{~d} t}$, and $\ddot{x}$ denotes $\frac{\mathrm{d}^{2} x}{\mathrm{~d} t^{2}}$.

\section{ENERGY SHAPING IN PBC}

We investigate whether it is possible for PBC to be applied to a system of multiple inverters coupled with dissipation. The stored energy of a circuit is the sum of the energy in the passive elements, that is the inductors and capacitors. The energy of the multiple inverter system is given by Eq.(16).

$$
H=\frac{1}{2} \boldsymbol{x}^{T} \mathbf{D} \boldsymbol{x}
$$

$\boldsymbol{x}$ and $\mathbf{D}$ are as given in Eqs.(8). $H$, which is the total energy which is a summation of the kinetic and potential energy.

The modified energy function is based on the Hamiltonian. The formulation is taken to be the quadratic function of errors. It is given by Eq.(17).

$$
H_{d}=\frac{1}{2} \boldsymbol{e}^{T} \mathbf{D} \boldsymbol{e}, \quad \boldsymbol{e}=\boldsymbol{x}-\boldsymbol{x}_{d}
$$

Here, $\boldsymbol{x}_{\boldsymbol{d}}$ is the desired trajectory of the state. In order to prove that this function is a candidate of Lyapunov function, let us check the derivative of the energy function as in Eq.(18).

$$
\begin{aligned}
\dot{H}_{d} & =\left(\boldsymbol{x}-\boldsymbol{x}_{d}(t)\right)^{T} \mathbf{D}\left(\dot{\boldsymbol{x}}-\dot{\boldsymbol{x}}_{d}(t)\right) \\
& =\left(\boldsymbol{x}-\boldsymbol{x}_{d}(t)\right)^{T}\left([\mathbf{J}-\mathbf{R}] \boldsymbol{x}+\mathbf{E}-\mathbf{D} \dot{\boldsymbol{x}}_{d}(t)\right)
\end{aligned}
$$

By setting the term $\mathbf{D} \dot{\boldsymbol{x}}_{d}(t)$ as given in Eq.(19), the control rule with PBC can be formulated for nuck-type inverters.

$$
\mathbf{D} \dot{\boldsymbol{x}}_{d}(t)=(\mathbf{J}-\mathbf{R}) \boldsymbol{x}_{d}(t)+\mathbf{E}+\mathbf{R}_{I}\left(\boldsymbol{x}-\boldsymbol{x}_{d}(t)\right)
$$

Where, $\mathbf{R}_{I}$ is a symmetric and positively defined matrix, which acts as the damping injection, making the system asymptotically stable if $\mathbf{R}+\mathbf{R}_{I}>0$. By considering that $\boldsymbol{e}^{T} \mathbf{J}(\mu) \boldsymbol{e}=0$ for all values of $\mu$, we get the following condition on the derivative of our chosen Lyapunov function $H_{d}(\boldsymbol{e})$.

$$
\dot{H}_{d}(e)=\boldsymbol{e}^{T}\left(\mathbf{J}(\mu) \boldsymbol{e}-\mathbf{R} \boldsymbol{e}-\mathbf{R}_{I}\right) \boldsymbol{e}=-\boldsymbol{e}^{T}\left(\mathbf{R}+\mathbf{R}_{I}\right) \boldsymbol{e}<0
$$

Under the control rule, the error $\boldsymbol{e}$ converges to the origin asymptotically. Then, by satisfying the condition $\left(\mathbf{R}+\mathbf{R}_{I}\right)>0$, the system becomes exponentially and asymptotically stable at the equilibrium point [29]. Then the derivative of $H_{d}(e)$ is given in Eq.(21).

$$
\dot{H}_{d}(\boldsymbol{e})=-\boldsymbol{e}^{T}\left(\mathbf{R}+\mathbf{R}_{I}\right) \boldsymbol{e} \leq-k H_{d}(\boldsymbol{e})
$$

Hence it is proved that Lyapunov's Theorem is satisfied. That is, the equilibrium state $\boldsymbol{x}_{d}$ is asymptotically stable with the control rule given in Eq.(19), and exponentially asymptotically stable with the damping injection. $\mathbf{R}_{I}$ is given in Eq.(22).

$$
\mathbf{R}_{\boldsymbol{I}}=\left[\begin{array}{cccc}
\mathbf{R}_{\boldsymbol{I 0}} & 0 & \ldots & 0 \\
0 & \mathbf{R}_{\boldsymbol{I} \mathbf{1}} & \ldots & 0 \\
\vdots & \vdots & \ddots & \vdots \\
0 & 0 & \ldots & \mathbf{R}_{\boldsymbol{I n}}
\end{array}\right] \text {, where } \mathbf{R}_{\boldsymbol{I n}}=\left[\begin{array}{ccc}
R_{\alpha \mathrm{n}} & 0 & 0 \\
0 & 0 & 0 \\
0 & 0 & 0
\end{array}\right]
$$




$$
\begin{aligned}
\dot{H}_{d}(e) & =\boldsymbol{e}^{T}(\mathbf{J}(\mu)) \boldsymbol{e}-\mathbf{R} \boldsymbol{e}-\mathbf{R}_{I} \boldsymbol{e} \\
& =-\boldsymbol{e}^{T}\left(\mathbf{R}+\mathbf{R}_{I}\right) \boldsymbol{e}<0
\end{aligned}
$$

For a buck-type inverter system, an indirect output stabilization is ensured by setting the current $\left(\bar{i}_{L}\right)$ to a constant sinusoidal value, depending on the desired output voltage amplitude and frequency. The zero dynamics of the bucktype inverter with respect to the output capacitor voltage being unstable, a feedback control is considered through current (indirect stabilization)[25]. Thus, we set a constant desired current, and then formulate the desired state values for the output voltage and dissipation current. The desired current $\left(\bar{i}_{L \mathrm{n}}\right)$ is obtained from the steady state analysis given by Eq.(14). The control equations are formulated based on the PBC equation Eq.(25)-(27).

$$
\begin{aligned}
\mu_{\mathrm{n}} & =\left[L_{\mathrm{n}} \dot{\bar{i}}_{L \mathrm{n}}+\tilde{v}_{C \mathrm{n}}-R_{\alpha}\left(i_{L \mathrm{n}}-\bar{i}_{L \mathrm{n}}\right)\right] \\
C_{\mathrm{n}} \dot{\tilde{v}}_{C \mathrm{n}} & =\bar{i}_{L \mathrm{n}}-\tilde{i}_{T \mathrm{n}}+\tilde{i}_{T \mathrm{n}-1}-\left[\frac{\tilde{v}_{C \mathrm{n}}}{R_{2 \mathrm{n}-1}}\right] \\
L_{T \mathrm{n}} \dot{\tilde{i}}_{T \mathrm{n}} & =\tilde{v}_{C \mathrm{n}}-\tilde{v}_{C \mathrm{n}+1}-R_{1 \mathrm{n}} \tilde{i}_{T \mathrm{n}}
\end{aligned}
$$

Here, $\boldsymbol{x}_{d}=\left[\begin{array}{lll}\bar{i}_{L} & \tilde{v}_{C} & \tilde{i}_{L T}\end{array}\right]^{\mathrm{T}}$ is the desired dynamic state vector for the controller, and $\mu$ is the feedback duty ratio.

The value of the duty ratio $\mu$ is evaluated atevery instant $t$ depending on the input, the parameters, and the desired output of the system. That is, $\mu$ depends on time and the state. Hereafter, when PBC is applied to the system, $\mu$ is considered as a function of time and state of the system.

To synchronize the phase of the output voltages of ring coupled buck-type inverter systems, the \#p inverter is taken as a reference. The sinusoidal reference for the \#p inverter is used as the input signal for the PLL, the output of which is used as the external frequency input for the sinusoidal reference of the $\# \mathrm{p}-1$ and $\# \mathrm{p}+1$ inverter. The sinusoidal reference of $\# \mathrm{p}-1$ and $\# \mathrm{p}+1$ is in turn used as the input to PLL, the output of which is used as the external frequency to $\# \mathrm{p}-2$ and $\# \mathrm{p}+2$ respectively. For odd number of inverters in the system, it ensures minimun time delay in synchronising the phase and frequency of all the inverters in the ring. The connection of the PLL to the system is shown in Fig.4.

\section{NUMERICAL SIMULATIONS}

The simulation results are obtained for five inverters coupled in a ring form are given in this section. The numerical simulations were carried out on ode45 solver Simulink (Version 8.7 R2016a). The numerical simulations are performed in three cases depending on the phase angle and frequency of the inverters in the ring.

\section{A. Case I: $\Delta \omega_{\mathrm{n}}=0, \theta_{\mathrm{n}}=0$}

In Case I we test the effectiveness of the original system as well as the system with PBC under balance and unbalanced conditions. There is no phase diference between the output of the inverter systems and the desired frequency remains constant at the centre frequency of the VCO. Thus $\omega_{i n}=\omega_{d n}=\omega_{d}$. This implies the output frequency of all inverters stays constant at a particular value.

The simulations were proceeded for a ring-coupled system consisting of 5 buck-type inverters to enhance the asymmetry in the system. Firstly, we investigate the effectiveness of the application of a sinusoidal averaged duty cycle to the switches through PWM to achieve AC voltage. Fig.5 shows system behaviour with the steady state analysis as given in Eq.(15). The steady state analysis is applied to a balanced ring coupled inverter system. A balanced system implies that the parameters of all inverter systems are set to be the equal. The parameters are given in Fig.3.

It is seen in Fig.5 that AC output voltage is generated in all five inverters, and due to the condition of balance, all inverters synchronize with each other. The energy of the entire system is the sum of the energy in the inductors (analogous to kinetic energy in mechanical systems) and the energy in the capacitors (analogous to the potential energy in mechanical systems). The energy of the system depends on the inductor currents and capacitor voltage, it oscillates with twice the frequency as the output voltage frequency. As all the inverter system and the dissipation between them is balanced, there is no flow of energy between the coupled inverters. 

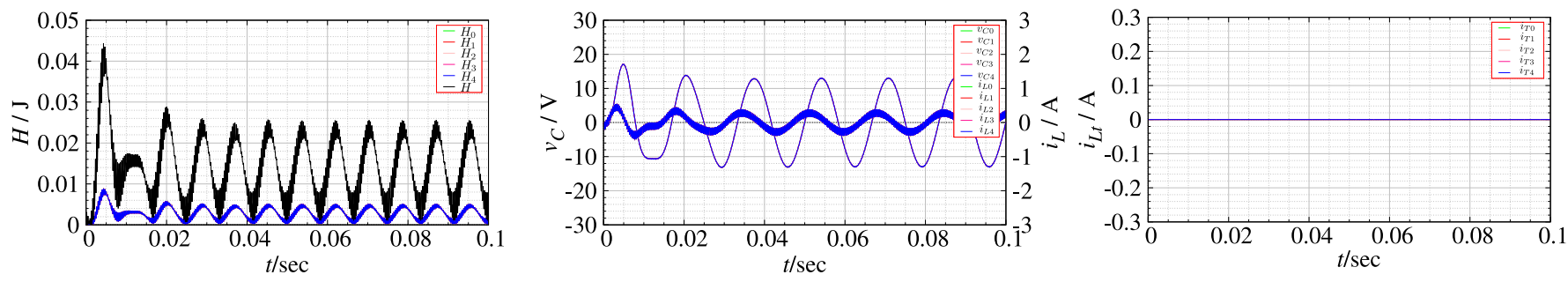

FIG. 5: Time evolution of original AC grid system without control. Here, $\bar{v}_{C \mathrm{n}}$ is set with parameters $A_{\mathrm{n}}=13$, $f_{\mathrm{n}}=60 \mathrm{~Hz}$.
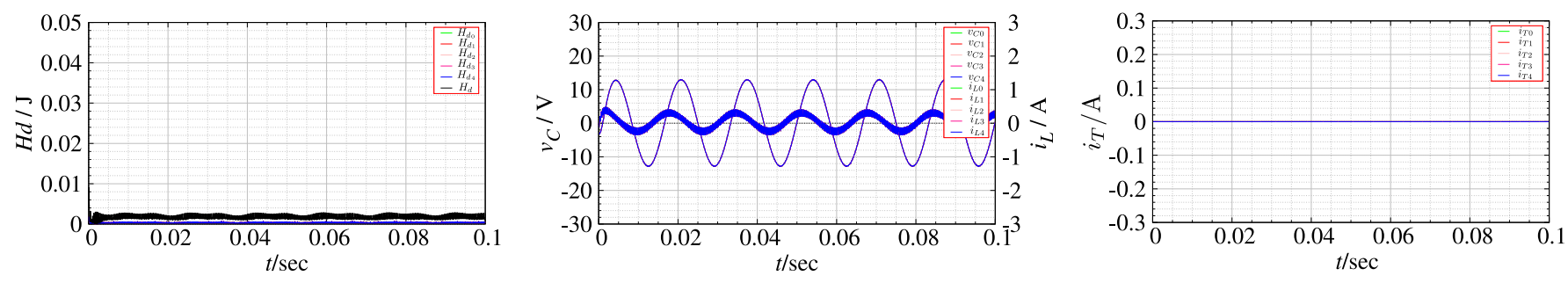

FIG. 6: Time evolution of AC grid with PBC. Here, $\bar{v}_{C \mathrm{n}}$ is set with parameters $A_{\mathrm{n}}=13, f_{\mathrm{n}}=60 \mathrm{~Hz}$.

Next, the system behaviour with the application of PBC is shown in Fig.6. The results show a marked improvement in the transient period of the dynamic behaviour, with a smaller transient peak amplitude and a faster convergence to the desired state. The behaviour of the designed energy function $\left(H_{d}\right)$ is also shown. The system achieves control when the value of the energy function drops down to zero. As the system output is not constant with time, the energy function oscillates around the zero value.

The network is said to be imbalanced when the coupled inverters in the ring have different parameters. For practical cases where the input comes from PV arrays, imbalance occurs at the change of input voltage for each inverter system, depending on the irradiation, and with the change in the resistances owing to changing loads. Therefore, next, we check the effectiveness of PBC by applying extreme noise to the input voltage as well as the load resistances. This will create high imbalance conditions, as the input voltage and load resistances for each inverter will change separately from the others. When the input voltage or load resistances are varied, the other parameters are kept consistent with the balanced case. The application of PBC to such an unbalanced system is shown in Fig.7 and Fig.8 for different input voltage and load transients respectively. It can be seen that despite extreme variation from the balanced values, the output is fairly sinusoidal in both the cases. The frequency as well as the phase is maintained. The transient expected at the sudden change of parameters is dampened and the output is kept fairly constant at the desired sinusoidal voltage. Imbalance occurring in the ring coupled inverter system creates a difference in the output voltage of the inverters, inducing the flow of dissipation current between the inverters.

\section{B. Case II: $\Delta \omega=0, \theta_{i \mathrm{n}}=$ constant}

In the numerical simulations shown in Sec.VII A, the frequency and phase of the output voltages was same for all the inverters. It was assumed that the sinusoidal reference for the PWM for all the inverters has the same frequency and is switched $\mathrm{ON}$ at the same time, resulting in zero phase difference between the outputs. Even though all the sinusoidal references can be provided with an external frequency, it is not fair to assume that all the sinusoidal references will start at the exact same moment. Thus, it is possible that there might be a phase difference between the sinusoidal references, and as a consequence, between the output voltages themselves.

Figures 9 and 10 show the output voltage and input currents of all 5 inverter systems without and with PBC respectively. The origin of the temporal axis is when all the input voltages are switched ON. For the numerical simulations \#0 is used as the reference inverter. The proportional gain $\left(K_{p}\right)$ of the PLL is set at 180 . To emulate a practical case, all the sinusoidal signals start at different times. Without PLL, it would create a constant phase difference in the outputs, leading to an undesirable output voltage. Here, \# 0 is considered as the reference inverter. The phase of this reference is as $\Phi=\omega_{d} t+0.6$, with an arbitarary constant phase angle $\theta_{i}=0.6 \mathrm{rad}$. It is observed that until all the sinusoidal references are switched ON, the output in unstable. As the reference signals are switched ON, the PLL achieves the lock-in condition and the system output settles down to the desired state. The centre 

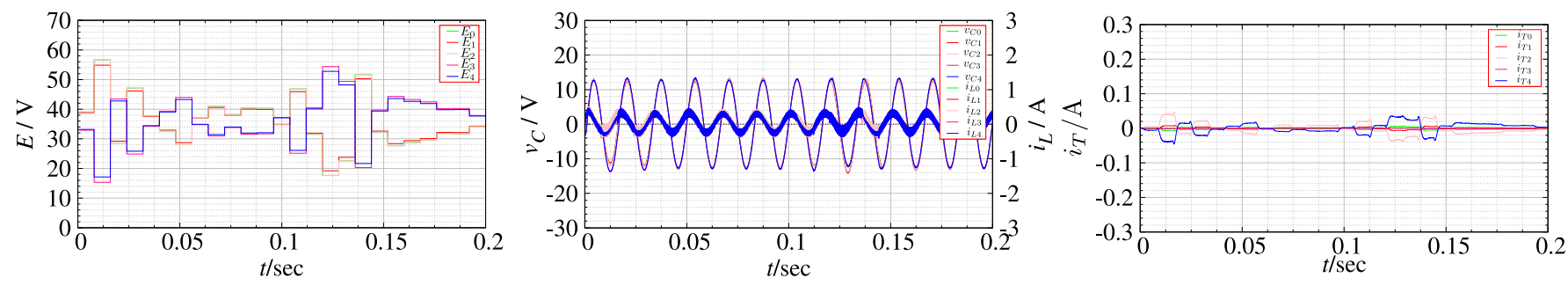

FIG. 7: Behaviour of unbalanced AC-grid with varying input voltages under PBC.
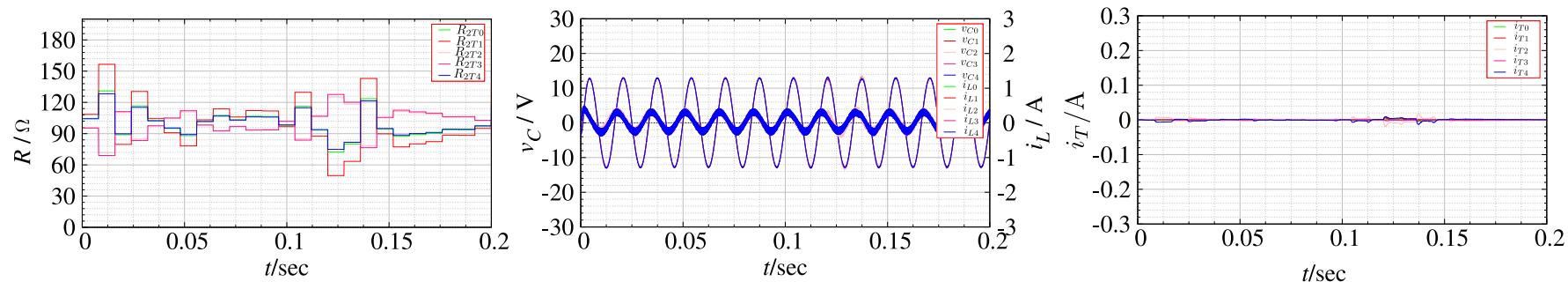

FIG. 8: Behaviour of unbalanced AC-grid with varying load resistances under PBC.

frequency is $\omega_{d}=377 \mathrm{rad} / \mathrm{sec}(60 \mathrm{~Hz})$.

Comparing the two figures, it is seen that the inverter output is more synchronized when PBC is applies, which is indicated by the dissipation current flowing between the inverters. The dissipation current for the original system is almost three times higher than when PBC is applied. The output settles down to the desired state faster with PBC, and the dissipation current goes to zero for all inverters. With $\mathrm{PBC}$, the duty ratio governing the output voltage dynamically changes to drive the energy function to the minimum value. The dynamic change in the duty ratio ensures a smaller deviation from the desired state due to the thorough consideration of the energy characteristics of the system through PBC. In both cases, the PLL shows the phase synchronization of all the inverters to that of the reference inverter.

\section{Case III: $\Delta \omega \neq 0, \theta_{i \mathrm{n}}=f(t)$}

Next we vary the desired frequency from the centre frequency of the oscillator frequency creating an error frequency $\Delta \omega=\omega_{i}-\omega_{d}$. Then, the tracking of the PLL is observed for changes in frequency. The frequency is varied in two ways. First, the frequency of this input is set to be different than the centre frequency of the VCO. For a VCO centre frequency of $377 \mathrm{rad} / \mathrm{sec}$, we set the initail input frequency to be $396 \mathrm{rad} / \mathrm{sec}$. Then, the frequency is varied linearly as a function of time with $\Delta \omega=10 t$. The results for these two cases is shown in Fig.11. Here, $\Delta \alpha$ is the difference between the sinusoidal signal of the reference inverter and the other inverters in the ring. Thus, $\Delta \alpha$ indicates the deviation of the PLL output from the desired sinusoidal reference. Note that $v_{e}=0$ for the purpose of numerical simulations.

The PLL achieves a phase lock in when the frequency is set at a different value from the centre frequency. After initial transient oscillations of different phase, the output steadies at the input frequency. Then, for the linear change in frequency, the PLL is able to keep the locked-in conditions even when the frequency is slowly increasing. The PLL maintains the hold-in conditions for the linearly increasing input frequency and retains all inverter systems at the input frequency. Thus, the PLL is successful in tracking the input successfully for these two cases of frequency change.

For cases where the frequency changes sinusoidally, the output shows an interference effect and the PLL may not be able to synchronize all the inverter outputs at the desired frequency. Thus a sinusoidal change in the frequency poses as a limitation of the synchronization with PLL. 

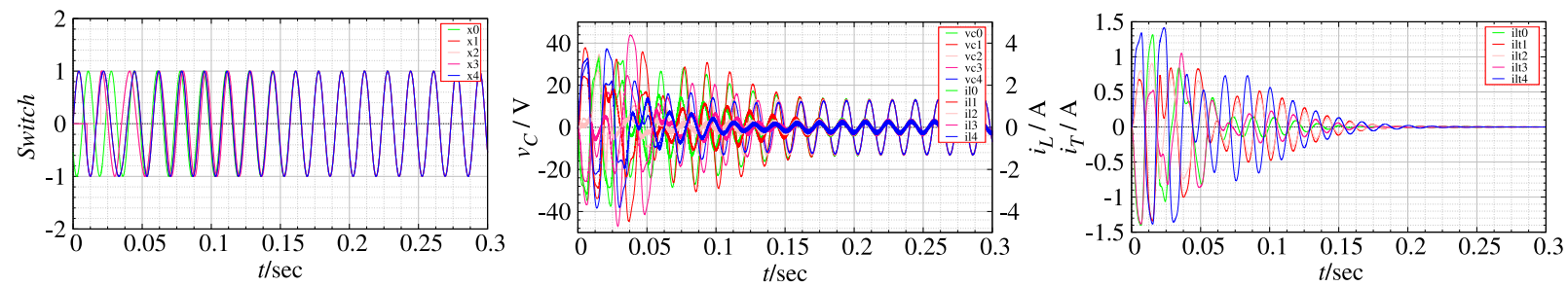

FIG. 9: Phase synchronization with PLL applied to autonomous AC grid of ring coupled buck-type inverter without PBC.
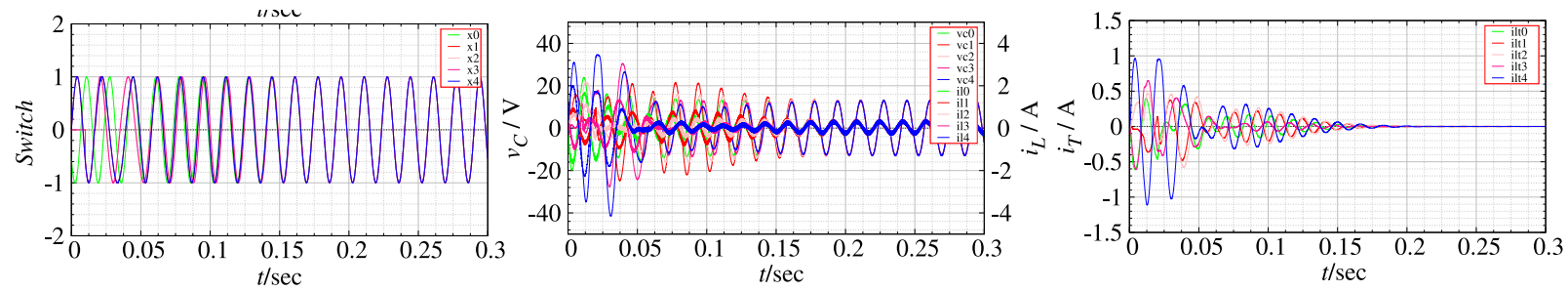

FIG. 10: Phase synchronization with PLL applied to autonomous AC grid of ring coupled buck-type inverter with PBC.
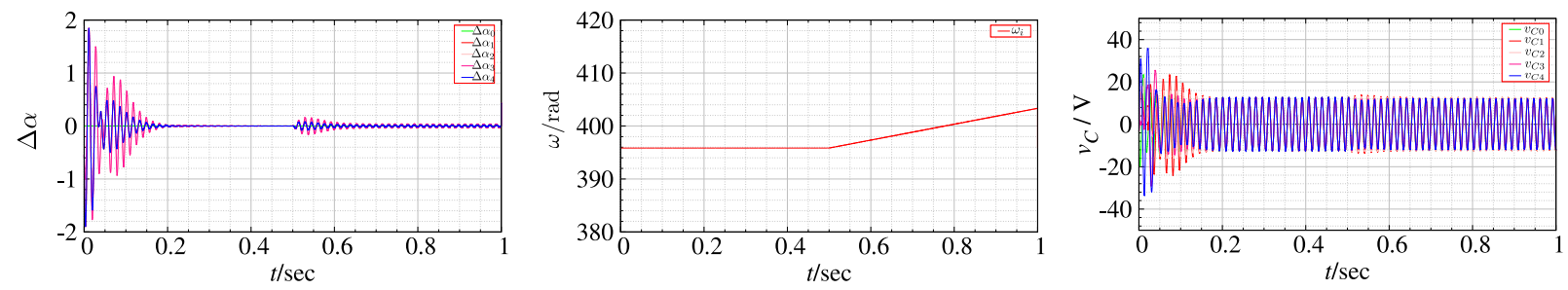

FIG. 11: Linear change in the frequency of the sinusoidal PWM reference.

\section{CONCLUSION}

In this paper we introduce an autonomous distributed generation AC grid to harness renewable DC sources of electricity. System stabilization for the grid to the desired output voltage is achieved through PBC. PBC with energy shaping and damping injection techniques was discussed with a modified energy function based on the quadratic function of errors. The modified function approaches zero as the system reaches the equilibrium condition.

A buck-type inverter was introduced to obtain a $\mathrm{AC}$ voltage with the desired amplitude and frequency by alternating the polarity of the DC input with the help of power electronic switches. Analysing the steady state of the system, the necessary switching is determined via the sinusoidal duty ratio. The control of the output voltage in a ring coupled inverter system was attained with the focus on the energy characteristics of the system with the application of PBC. The control equations were obtained by proving the desired energy function as a Lyapunov function. The convergence to the desired steady state as well as the effectiveness of PBC were verified by numerical simulations. The system was studied under conditions of imbalance. Numerical simulations indicate that PBC proves to remain an effective method of control under imbalance conditions.

It was found that phase differences between the output voltage of different inverter systems leads to unstable output voltage for the entire system. PBC causes the system to converge to a desired state, but even small inconsistencies in frequency can prove to be detrimental. In order to avoid such instabilities we implement frequency and phase angle synchronization to the entire system through PLL. Application of PLL was achieved through mathematical modelling, and then studied through numerical simulations. It was seen that the PLL successfully tracks the input reference for frequencies other than the centre frequency for the VCO as well as a linear change in the input frequency. Thus, a PLL, along with PBC, proves to be an efficient method to bring about phase synchronization in an autonomous grid with ring coupled buck-type inverters. 
[1] L. S. Thomas Ackermann, Goran Andersson, "Distributed generation: a definition," Electric Power System Research, vol. 57, no. 3, pp. 195-204, 2001.

[2] T. Adefarati and R. Bansal, "Integration of renewable distributed generators into the distribution system: a review," IET Renewable Power Generation, vol. 10, no. 7, pp. 873-884, 2016.

[3] A. Castillo and D. F. Gayme, "Grid-scale energy storage applications in renewable energy integration: A survey," Energy Conversion and Management, vol. 87, pp. 885-894, 2014.

[4] Q.-C. Zhong and T. Hornik, Control of power inverters in renewable energy and smart grid integration, vol. 97. John Wiley \& Sons, 2012.

[5] R. K. Akikur, R. Saidur, H. W. Ping, and K. R. Ullah, "Comparative study of stand-alone and hybrid solar energy systems suitable for off-grid rural electrification: A review," Renewable and sustainable energy reviews, vol. 27, pp. 738-752, 2013.

[6] S. Goel and R. Sharma, "Performance evaluation of stand alone, grid connected and hybrid renewable energy systems for rural application: A comparative review," Renewable and Sustainable Energy Reviews, vol. 78, pp. 1378-1389, 2017.

[7] M. Patterson, N. F. Macia, and A. M. Kannan, "Hybrid microgrid model based on solar photovoltaic battery fuel cell system for intermittent load applications," IEEE Transactions on Energy Conversion, vol. 30, no. 1, pp. 359-366, 2014.

[8] A. S. Anees, "Grid integration of renewable energy sources: Challenges, issues and possible solutions," in 2012 IEEE 5th India International Conference on Power Electronics (IICPE), pp. 1-6, IEEE, 2012.

[9] P. Acharjee, "Strategy and implementation of smart grids in india," Energy Strategy Reviews, vol. 1, no. 3, pp. 193-204, 2013.

[10] P. Loka, S. Moola, K. Polsani, S. Reddy, S. Fulton, and A. Skumanich, "A case study for micro-grid pv: lessons learned from a rural electrification project in india," Progress in Photovoltaics: Research and Applications, vol. 22, no. 7, pp. 733-743, 2014.

[11] A. Kanase-Patil, R. Saini, and M. Sharma, "Integrated renewable energy systems for off grid rural electrification of remote area," Renewable Energy, vol. 35, no. 6, pp. 1342-1349, 2010.

[12] J. G. Kassakian, M. F. Schlecht, and G. C. Verghese, Principles of power electronics. Graphis, 2000.

[13] Y. Miladi, M. Feki, and N. Derbel, "Optimal control of a single-phase h-bridge dc-ac inverter," International Journal of Circuit Theory and Applications, vol. 44, no. 3, pp. 744-758, 2016.

[14] A. Tofighi and M. Kalantar, "Power management of pv/battery hybrid power source via passivity-based control," Renewable Energy, vol. 36, no. 9, pp. 2440-2450, 2011.

[15] P. L. Jie Bao, Process Control- The Passive Systems Approach. Springer-Verlag, 2007.

[16] R. Ortega, J. A. L. Perez, P. J. Nicklasson, and H. Sira-Ramirez, Passivity-based control of Euler-Lagrange systems: mechanical, electrical and electromechanical applications. Springer Science \& Business Media, 2013.

[17] R. Ortega, A. Van der Schaft, B. Maschke, and G. Escobar, "Energy-shaping of port-controlled hamiltonian systems by interconnection," in Decision and Control, 1999. Proceedings of the 38th IEEE Conference on, vol. 2, pp. 1646-1651, IEEE, 1999.

[18] C. Batlle, A. Dòria-Cerezo, and E. Fossas, "Bidirectional power flow control of a power converter using passive hamiltonian techniques," International journal of circuit theory and applications, vol. 36, no. 7, pp. 769-788, 2008.

[19] H. Sira-Ramirez and R. Ortega, "Passivity-based controllers for the stabilization of dc-to-dc power converters," in Decision and Control, 1995., Proceedings of the 34th IEEE Conference on, vol. 4, pp. 3471-3476, IEEE, 1995.

[20] M. Zhu and F. L. Luo, "Transient analysis of multi-state dc-dc converters using system energy characteristics," International Journal of Circuit Theory and Applications, vol. 36, no. 3, pp. 327-344, 2008.

[21] T. Hikihara and Y. Murakami, "Regulation of parallel converters with respect to stored energy and passivity characteristics," IEICE transactions on fundamentals of electronics, communications and computer sciences, vol. 94, no. 3, pp. 1010-1014, 2011.

[22] N. Jaalam, N. Rahim, A. Bakar, C. Tan, and A. M. Haidar, "A comprehensive review of synchronization methods for grid-connected converters of renewable energy source," Renewable and Sustainable Energy Reviews, vol. 59, pp. 1471-1481, 2016.

[23] F. Blaabjerg, R. Teodorescu, M. Liserre, and A. V. Timbus, "Overview of control and grid synchronization for distributed power generation systems," IEEE Transactions on industrial electronics, vol. 53, no. 5, pp. 1398-1409, 2006.

[24] G.-C. Hsieh and J. C. Hung, "Phase-locked loop techniques. a survey," IEEE Transactions on industrial electronics, vol. 43, no. 6, pp. 609-615, 1996.

[25] H. Sira-Ramírez and R. Silva-Ortigoza, Control design techniques in power electronics devices. Springer Science \& Business Media, 2006.

[26] R. E. Best, Phase locked loops: design, simulation, and applications. McGraw-Hill Professional, 2007.

[27] M. Karimi-Ghartema, Enhanced phase-locked loop structures for power and energy applications. John Wiley \& Sons, 2014.

[28] A. Van Der Schaft, "Port-hamiltonian systems: network modeling and control of nonlinear physical systems," in Advanced dynamics and control of structures and machines, pp. 127-167, Springer, 2004.

[29] H. K. Khalil, "Noninear systems," Prentice-Hall, New Jersey, vol. 2, no. 5, pp. 5-1, 1996. 


\section{APPENDIX A: PHASE LOCKED LOOP}

An inconsistency in the frequency or phase of the output voltages causes an interference effect. This effect is not desirable as the output voltage amplitude becomes unstable, instead of a pure sinusoidal waveform. Frequency as well as phase synchronization can be achieved with the help of a phase locked loop.

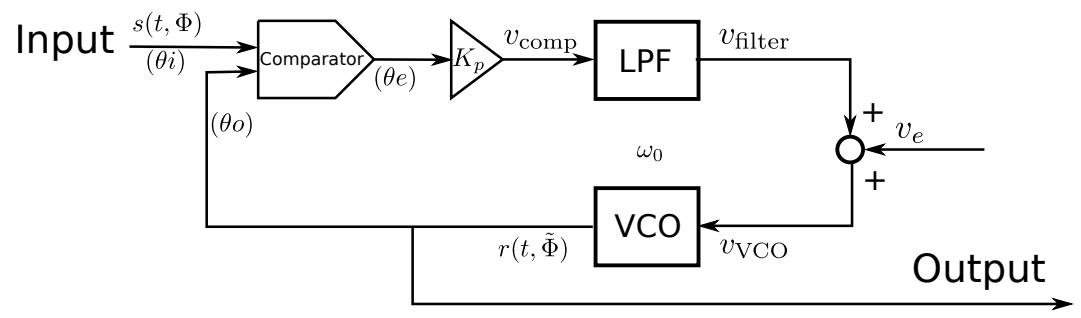

FIG. 12: Block diagram of a phase locked loop.

A phase locked loop is a circuit that generates an output signal, whose phase is the same as that of the input signal [24]. A PLL generally consists of a phase detector or a comparator, a VCO and a low pass filter [26, 27]. The phase detector(PD) produces an error signal that is proportional to the phase error between the input signal and the output of the VCO. The low pass filter supresses noise and the unwanted PD outputs, thus determining the dynamics of the PLL. The VCO produces a signal with frequency proportional to the control voltage $\left(v_{\mathrm{VCO}}\right)$. The closed loop forces the VCO to lock the phase of the output to that of the input signal. The output of the VCO is the output of the PLL. The block diagram of a phase locked loop is shown in Fig.12.

The input signal to the PLL is the sinusoidal signal for the PWM of the reference inverter. Let the input signal be given by $s(t, \Phi)=A \sin \left(\omega_{d} t+\theta_{i}(t)\right)$, where $A$ is the amplitude and $\phi=\omega_{d} t+\theta_{i}(t)$ is the phase of the input signal. $\omega_{d}$ is the centre frequency of the VCO. $\theta_{i}(t)$ incorporates the error in the input frequency, $\delta \omega=\omega_{i}-\omega_{d}$ from the centre frequency of the VCO. Let the output of the VCO be $r(t, \tilde{\Phi})=V_{0} \cos \left(\omega_{d} t+\theta_{o}(t)\right)$, with $V_{0}$ as the amplitude and $\tilde{\Phi}=\omega_{d} t+\theta_{o}(t)$ as the phase.

With $s(t, \Phi)$ and $r(t, \tilde{\Phi})$ as the inputs to the PD, the output voltage of the PD can be given by Eq.(28).

$$
v_{\text {comp }}=K_{p} A \sin \left(\theta_{e}\right)
$$

Here, $K_{p}$ is the gain from the $\mathrm{PD}$ and $\theta_{e}=\theta_{i}-\theta_{o}$ is the phase error. It is set a dimensionless quantity and is set at $K_{p}=A V_{0} / 2 . v_{\text {comp }}$ is the input of the LPF. If the transfer function of the LPF in the frequency domain is given as $F(s)$, then the output voltage of the filter can be obtained by Eq.(29). The LPF is considered ideal without any harmonics.

$$
v_{\text {filter }}=\mathcal{L}^{-1}\left[F(s) V_{\text {comp }}(s)\right]
$$

Here, $\mathcal{L}^{-1}$ denotes the Laplace inverse and $V_{\text {comp }}(s)$ is the Laplace transform of $v_{\text {comp. }}$. The instantaneous output frequency of the $\mathrm{VCO}$, given by $\omega_{\mathrm{VCO}}(t)$, is proportional to the control input of the VCO. Then, Eq.30 can be obtained.

$$
\omega_{\mathrm{VCO}}(t)=\omega_{d}+K_{C} v_{\mathrm{VCO}}
$$

Here, $K_{C}$ represents the gain of the VCO. The units are $\mathrm{Hz} / \mathrm{V}$ and is calculated as $f_{\max }-f \min / v_{\max }-v_{\min }$. Where $f_{\max }$ and $f_{\min }$ represent the maximum and minimum frequency, and $v_{\max }$ and $v_{\min }$, the maximum and minimum voltage respectively. The instantaneous frequency of of the output of the VCO is given by the derivative of $\tilde{\Phi}$. Thus, the following set of equations hold, where $v_{e}$ is the external control voltage.

$$
\begin{aligned}
\omega_{\mathrm{VCO}}(t) & =\frac{d \tilde{\Phi}}{d t} \\
\frac{d \theta_{o}}{d t} & =K_{C} v_{\mathrm{VCO}} \\
v_{\mathrm{VCO}} & =v_{\text {filter }}+v_{e}
\end{aligned}
$$


Thus, the phase of the output voltage, $\tilde{\Phi}$ is locked with the phase of the input, and the output signal can be used as a sinusoidal reference to the PWM of the adjoining inverter. The loop equation for $\theta_{o}(t)$ is given by Eq.(34)

$$
\frac{d \theta_{o}}{d t}=K_{C}\left[\mathcal{L}^{-1}\left[F(s) V_{\text {comp }}(s)\right] K_{p} A \sin \left(\theta_{e}\right)+v_{e}\right]
$$

Here, $K_{C}$ represents the gain of the VCO. The units are $\mathrm{Hz} / \mathrm{V}$ and is calculated as $f_{\max }-f \min / v_{\max }-v_{\min }$. Where $f_{\max }$ and $f_{\min }$ represent the maximum and minimum frequency, and $v_{\max }$ and $v_{\min }$, the maximum and minimum voltage respectively. 